\title{
Discrepancy Analysis of Load-Displacement in the Combination Space for Concrete Box Girder Assessment
}

\author{
X. F. Ye, ${ }^{\mathrm{a}, 1}$ H. Ogai, ${ }^{\mathrm{a}}$ and C. W. Kim ${ }^{\mathrm{b}}$ \\ ${ }^{a}$ Faculty of Science and Engineering, Waseda University, Tokyo, Japan \\ ${ }^{\mathrm{b}}$ Graduate School of Engineering, Kyoto University, Kyoto, Japan \\ ${ }^{1}$ yexiongfei@gmail.com (Xiong-Fei Ye)
}

The combination space concept had its origin in the theory of subspace and combinatorics. The combination space may be treated as the space reconstruction through combination, as well as the subspace one. Several new analytical methods are proposed based on combination principles applied to some operations. Under progressing structural damage, simple raw data cannot adequately characterize continuously changing processes and provide early prevention of considerable damage or loss of resistance to hazardous failure. Therefore, the data need to be further processed to find new criteria and factors of damage assessment. Three evaluation methods were experimentally established with the combination space. For the damage assessment and its early prevention some factors are also proposed such that the combination space method can find the structural change earlier than the original data space. In the case of the the disparity between different conditions and the initial state, this method can contribute to the structure recovery, preventing its failure.

Keywords: combination space, load-displacement, discrepancy analysis, concrete box girder.

Introduction. The load-displacement (stress-strain) analysis in the research of structural material is an old topic that through the change of the elastic modulus or elasticity coefficient, the change of the structure of general dimensional load-displacement will be intuitively demonstrated [1]; even in the fractal dimensional load-displacement, it can also be known [2]. In the view of damage mechanics [3, 4], the change of the structure has the growth of microcracks and cavities will lead the system to be dangerous [5]. The damage happened in the structure is because of the static-dynamic loading, heating/ temperature-changing, rust/corrosion, etc. [6].

On the other hand, for the existence of damage, the resistance of the structure will go down which can be an indicator in damage evaluation, this kind of research in the view of fixed relationship is named as the structural stress analysis [7], which can be modeled by the finite element method (FEM) for a complex system to find and evaluate the damage, and this kind of research in the view of probability is often named as the reliability research of structure [8]. In the evaluation of the local damages and the global damage, the local damages will influence the global damage, and often strength the damage of global. The investigation of local stiffness degradation can be used to detect the global damage [9]. Through FEM method, such kind of relationship will be more convenient to express [10].

Then, the damage development in the structural lifetime is from micro to macro, and then destroy the structure (it is an ideal situation, but it need be avoided). The displacement averaging can be used to show the change in this development [11]. In other words, this method uses the relationship of local change and global change, through the displacement averaging of a continuous system, the global damage is described, but it cannot be used for local damage detection. Some case concentrates the damage detection of the local damage [10] using discrete elements, but it is not proper for the global damage detection. For a more comprehensive understanding of the damage development in the structure, there is a 
need to development of local-global analysis. To propose a method for both local damage and global damage, a combination method will be used. This method is based on the combinatorics [12] and combinational analysis [13].

Since there is a notation for the combinational elements in this research is the subspace [14], through the combination of these spaces and subspaces, there is a chance to present new information for the structural damage detection. In this research, three kinds of combination space are constructed which shows the structural damage detection based on the combination space is efficient and sensitive. In the data analysis for experiments using combination-space-based methods, the curves compared with the curve of original data will also be obtained.

1. The Basic Concept and Research Method and Research Target. In the initial research, the combination space is just the linear combination space.

For a series, a vector or a series of vectors whose data describes/measures the same thing:

$$
\mathbf{x}=\left\{\mathbf{x}_{1}, \mathbf{x}_{2}, \mathbf{x}_{3}, \ldots, \mathbf{x}_{s}, \ldots, \mathbf{x}_{n}\right\}=\left\{\mathbf{x}_{s}\right\},
$$

in which $s=\{i, j, k, \ldots\}$, and $i, j, k, \ldots=\{1,2,3, \ldots, n\}$,

$$
\mathbf{x}_{\text {new }}=\text { operation }(\mathbf{x})
$$

For example, using the combinatorics, the operation "+", and its inverse operation "-", the original information can be transferred into the new series,

$\mathbf{x}_{\text {new }}=\ldots,\left\{-\mathbf{x}_{i}-\mathbf{x}_{j}-\mathbf{x}_{k}\right\},\left\{-\mathbf{x}_{i}-\mathbf{x}_{j}\right\},\left(-\mathbf{x}_{i}\right\},\{0\},\left\{\mathbf{x}_{i}\right\},\left\{\mathbf{x}_{i}+\mathbf{x}_{j}\right\},\left\{\mathbf{x}_{i}+\mathbf{x}_{j}+\mathbf{x}_{k}\right\}, \ldots$

as well as $\mathbf{x}_{\text {new }}=\{\}$ (the empty set). So, there are $1+2\left(n+n^{2}+n^{3}+\ldots\right)$ kinds of combinations. If the combination is limited by $m=M$, there is

$$
S_{n}= \begin{cases}1+2 \frac{n^{M+1}-n}{n-1}, & n \neq 1, \\ 1+2 M, & n=1 .\end{cases}
$$

For the same operation "+" in the combination space, there are

(i) for any two elements in combination space, the result of the operation of the two elements is also in this combination space;

(ii) for any three elements, they meet the law of associativity;

(iii) there is an element \{\} for any other element having an operation with this element, the result will be itself;

(iv) for any element, there exists another element that when having an operation with this element, the result is $\{0\}$.

So, it is a group. If it meets the law of commutation, it will be an Abel group.

In the damage detection for a concrete structure, three kinds of combination space will be used in this paper, i.e., combination structural information, information of difference/ similarity, and denominator information.

1) The combined structural information.

This kind of information will be obtained from $\mathbf{x}=\left\{\mathbf{x}_{1}, \mathbf{x}_{2}, \mathbf{x}_{3}, \ldots, \mathbf{x}_{i}, \ldots, \mathbf{x}_{n}\right\}$ that some data will be selected to describe some characteristic that

$$
\mathbf{x}_{j}=\left\{\mathbf{x}_{1, j}, \mathbf{x}_{2, j}, \mathbf{x}_{3, j}, \ldots, \mathbf{x}_{i, j}, \ldots, \mathbf{x}_{n, j}\right\}
$$

For example, $\mathbf{x}_{i, j}=\max \left(\mathbf{x}_{i}\right)$. 
2) The information of difference/similarity.

For $\mathbf{x}=\left\{\mathbf{x}_{1}, \mathbf{x}_{2}, \mathbf{x}_{3}, \ldots, \mathbf{x}_{i}, \ldots, \mathbf{x}_{n}\right\}$, there is a difference/similarity matrix:

$$
\rho=\left[\begin{array}{cccccc}
\rho_{(1,1)} & \rho_{(1,2)} & \cdots & \rho_{(1, j)} & \cdots & \rho_{(1, n)} \\
\rho_{(2,1)} & \rho_{2,2)} & \ldots & \rho_{(1, j)} & \ldots & \rho_{(2, n)} \\
\vdots & \vdots & \ddots & \vdots & \ddots & \vdots \\
\rho_{(i, 1)} & \rho_{(i, 2)} & \ldots & \rho_{(i, j)} & \cdots & \rho_{(i, n)} \\
\vdots & \vdots & \ddots & \vdots & \ddots & \vdots \\
\rho_{(n, 1)} & \rho_{(n, 2)} & \cdots & \rho_{(n, j)} & \cdots & \rho_{(n, n)}
\end{array}\right]=\left[\rho_{(i, j)}\right], \quad i, j=1,2, \ldots, n .
$$

3) The denominator information.

For $\mathbf{x}=\left\{\mathbf{x}_{1}, \mathbf{x}_{2}, \mathbf{x}_{3}, \ldots, \mathbf{x}_{i}, \ldots, \mathbf{x}_{n}\right\}$, this kind of information often refers to not only some operations,

$$
\mathbf{x}_{\text {new }}=\text { operations }(\mathbf{x})=f(\mathbf{x})
$$

but also some transforms after the operation,

$$
\mathbf{y}_{\text {new }}=\text { transforms }\left(\mathbf{x}_{\text {new }}\right)=g\left(\mathbf{x}_{\text {new }}\right) \text {, }
$$

like the Fourier, Hilbert, and Laplace transforms. So, for the second case, after the transform, there is

$$
\mathbf{y}_{\text {new }}=\left\{f\left(\mathbf{x}_{i}\right)\right\},\left\{f\left(\mathbf{x}_{i}+\mathbf{x}_{j}\right)\right\},\left\{f\left(\mathbf{x}_{i}+\mathbf{x}_{j}+\mathbf{x}_{k}\right)\right\}, \ldots
$$

2. Experiment, Analysis, and Result for Artificial Damage on One Box Girder. The experiment is to investigate the structural change when there is artificial damage, by the discrepancy analysis of different loading steps. This experiment object is near all-scale pre-stressed reinforced concrete open-section box girder (in short, box girder) in Public Works Research Institute (Fig. 1), with $8500 \mathrm{~mm}$ length, $2300 \mathrm{~mm}$ width, and $1000 \mathrm{~mm}$ height. On this girder, there are 4 SDP-200 of displacement meters, 8 SDP-100 of displacement meters (Table 1).

$\mathrm{T}$ a $\mathrm{b} 1 \mathrm{e} 1$

The Introduction of the Sensors

\begin{tabular}{|c|c|c|c|c|c||}
\hline Sensor & Type & Number & Purpose & \multicolumn{2}{|c|}{ Direction and location } \\
\hline $\begin{array}{c}\text { Displacement } \\
\text { meter }\end{array}$ & $200 \mathrm{~mm}$ & 4 & For deflection & Vertical & Under the girder \\
\cline { 2 - 6 } & $100 \mathrm{~mm}$ & 8 & For deflection & Vertical & $\begin{array}{c}\text { Both under and } \\
\text { up the girder }\end{array}$ \\
\cline { 2 - 6 } & $25 \mathrm{~mm}$ & 4 & $\begin{array}{c}\text { Subsidence } \\
\text { at the fulcrum }\end{array}$ & Vertical & Fulcrum of the girder \\
\hline
\end{tabular}

In the experimental process (Fig. 2), there are 384 steps in total (Fig. 3), and the sensors D3_F, D3_B, D4_F, D4_B can only test the step 1-360. For the data analysis, the data includes the array and matrix in three kinds of combinations. For the original data, using the style of the array, for every test step, some results like the average of the data, the root-mean-square will be obtained. The first category of the information is the combined structural information (Fig. 4). 

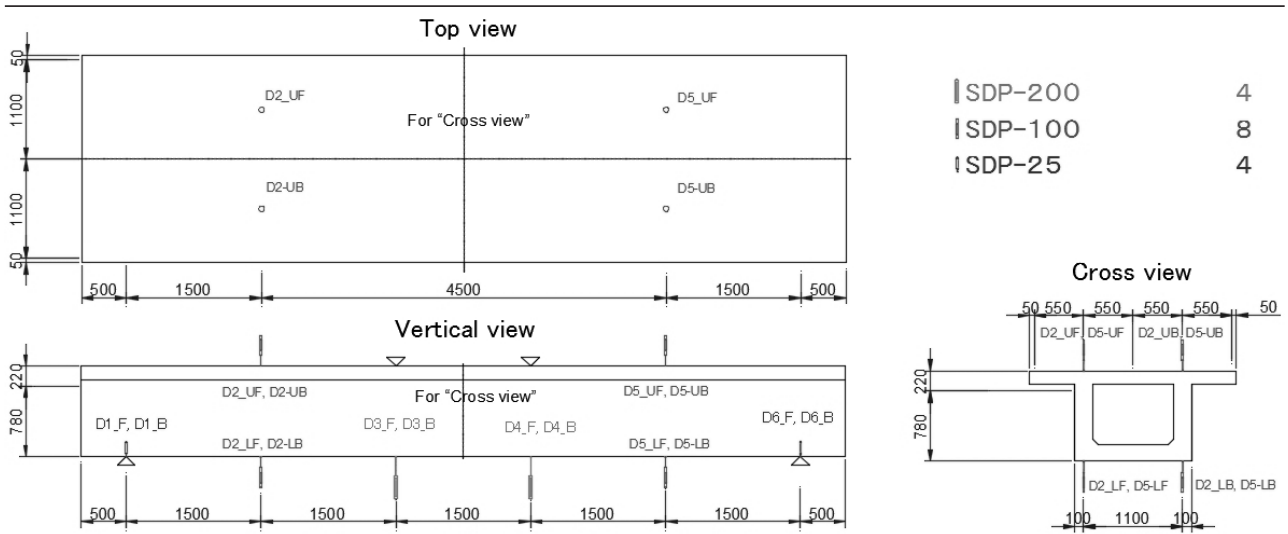

Fig. 1. The tested object, D1-D6 specify locations, while F and B mean "Front" and "Back", respectively.

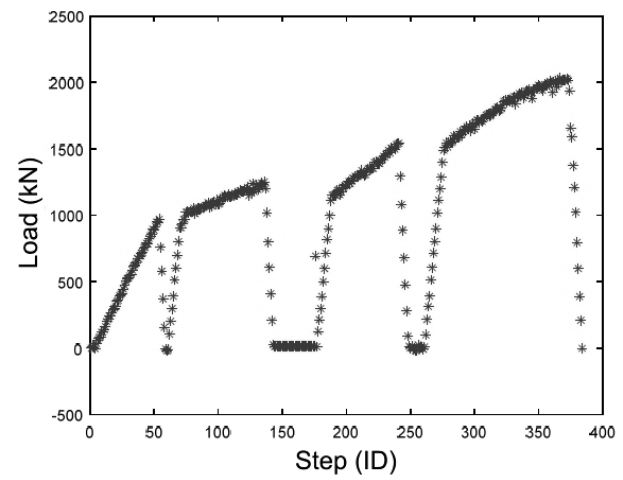

Fig. 2

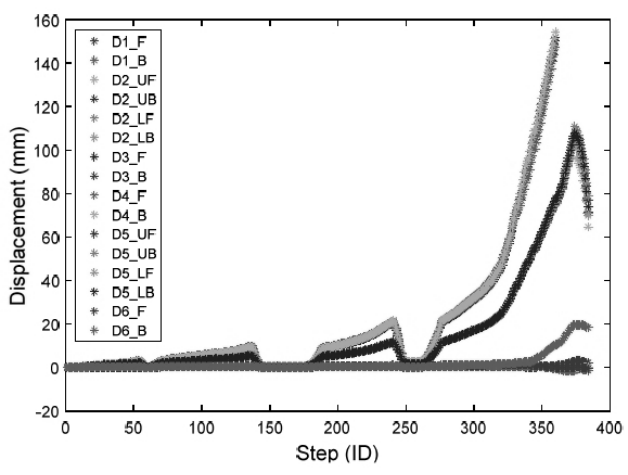

Fig. 3

Fig. 2. The loading process of this experiment.

Fig. 3. The experiment process of displacement of all meters from step 1-384.
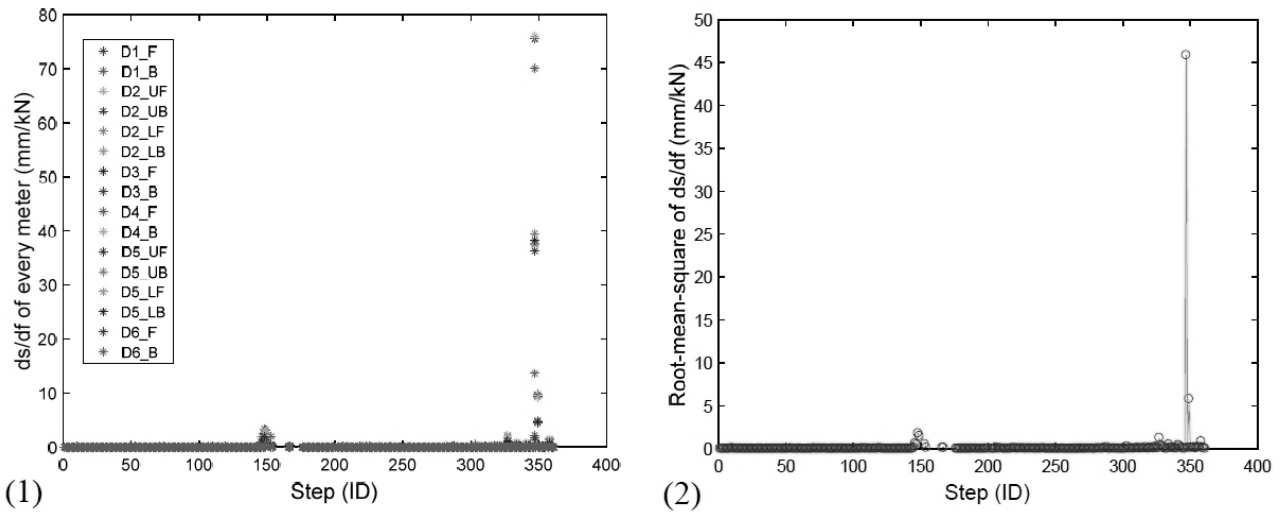

Fig. 4. (1) The result of differential derivations of every meter, according to Appendix 1; (2) the root-mean-square (all meters from step 1-360), according to Appendix 2.

From the loading process in Fig. 2 and data processing in Fig. 4 both sub-figure (1) of the differential derivations, and sub-figure (2) of the root mean square of the differential derivations that near the step 350 , there is a significant change, which can be used to 
indicate the most significant system change that there is a substantial damage in the structure.

For the second category of combination space of the information of difference/ similarity, compared with the original displacement, there are two kinds of combination spaces.

Even though Figs. 2 and 3 do not have the same tendency for all meters. But after the data processing, both shapes of figures (Fig. 5) of difference matrices' norm and distance are in the same trend with the loading process in Fig. 2 which can be used to directly show the system change evaluated by the difference matrix and the loading has the linear relationship.
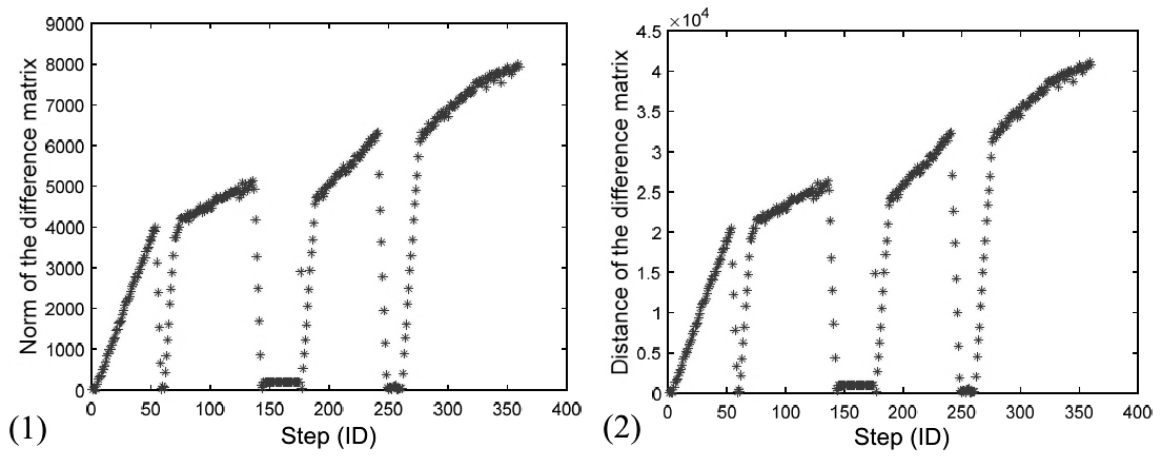

Fig. 5. (1) Norm of difference matrix, according to Appendix 3; (2) distance of difference matrices between considered and initial state (all meters from step 1-360), according to Appendix 4.
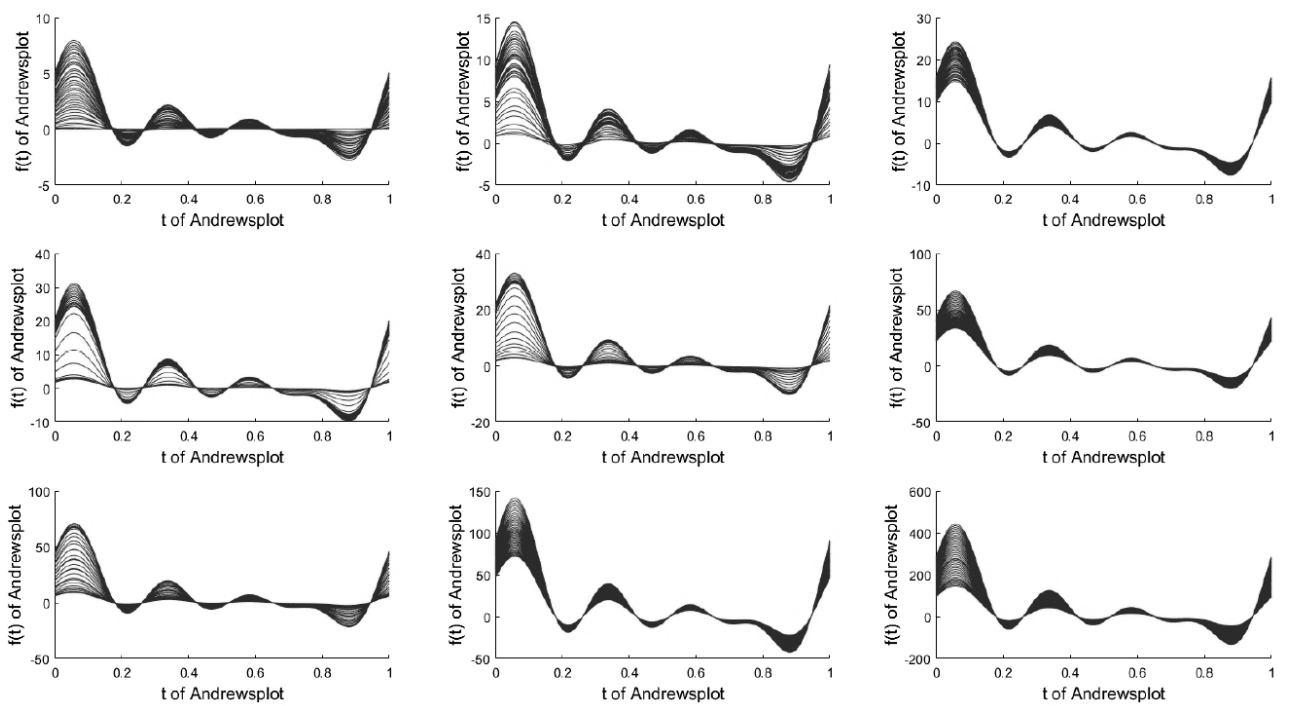

Fig. 6. Divide all 360 step into nine groups, from top to bottom, from left to right: step 1-40, step 41-80, step 81-120, step 121-160, step 161-200, step 201-240, step 241-280, step 281-320, and step 321-360.

For the third category, here is an example using Andrews plot/curve [16] for $[0,1]$ in Fig. 6, which is used to visualize high-dimensional data. Suppose $x=\left\{x_{1}, x_{2}, x_{3}, \ldots, x_{i}\right.$, $\left.\ldots, x_{n}\right\}$, the Andrews plot defines a finite Fourier series:

$$
f_{x}(t)=\frac{x_{1}}{\sqrt{2}}+x_{2} \sin (t)+x_{3} \cos (2 t)+x_{4} \sin (4 t)+x_{5} \cos (5 t)+\ldots
$$


From Fig. 6, one can see that in the statistics, from step 1 to step 360, the average of the $f(t)$ in the Andrews plot becomes greater in the first maximum. When there is some high damage, the extent of change for the Andrews plot will be greater. For more information, all figures have the same tendency which shows the data relationship between all meters are keep the same from the beginning to the end (from step 1 to step 360). For more discussion, here is a comparison between combination space and the subspace.

In contrast to the concept of combination space, there is a notion of subspace. Here is the definition of linear subspace [16]. Let $K$ be a field (such as the real numbers), $V$ be a vector space over $K$, and let $W$ be a subset of $V$. Then $W$ is a subspace if (1). The zero vector, 0 , is in $W$. If $u$ and $v$ are elements of $W$, then the sum $u+v$ is an element of $W$; (3) If $u$ is an element of $W$ and $c$ is a scalar from $K$, then the scalar product $c u$ is an element of $W$. In some sense, the combination space can also be treated as the combination of the subspaces since the general linear space can be interpreted as the union of all subspaces.
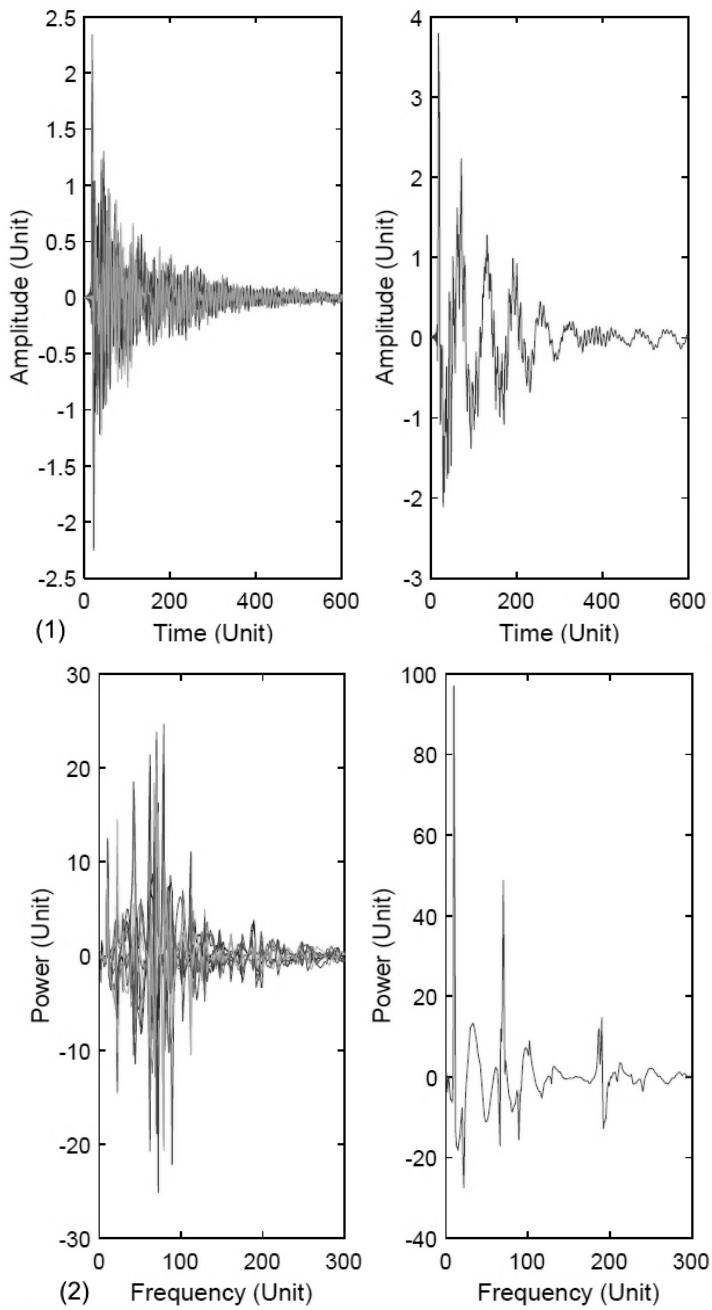

Fig. 7. The frequency using FFT for both general data space and combination space: (1) original data space in the form of multi-time series (left) vs combination space in the form of a sum of all time series (right); (2) the frequency obtained by FFT with original data space in the form of original multi-time series (left) and combination space in the form of a sum of all time series (right) separately. 
Moreover, in other application, for instance, the Fourier transform (fast Fourier transform, FFT) for records of acceleration sensors on the same structure as the response to the impulse action in Fig. 7. Even though some information, like some modes in stochastic subspace identification, will miss, but the stationary natural frequency of structure will be found.

Conclusions. The combination space can be treated as the information mining from kinds of general investigation, through the analysis of the relationship of those data to find some new information, from difference/similarity among kinds of general investigation, characteristics reconstruction, or transformation. In the linear space, the combination space can be regarded as the combination of not only the general space but also the combination of subspace through some operation and then its inverse operation.

By considering the structural damage and the combination space together, the data analysis of load-displacement can be investigated in 3 kinds as well, the difference matrices for the data of different locations, the average of data measured in different locations, and the transform of the data through Andrews plot. From the experiment result, through these combinations, it adequately reflects the continuous changing of the system and the early-warning for significant damage.

\section{Appendix 1.}

In each column, get new time series, $\mathbf{x}_{i, j}=\left(\mathbf{x}_{i+1, j}-\mathbf{x}_{i, j}\right) /\left(\mathbf{x}_{i, j}-\mathbf{x}_{i-1, j}\right)$, the result is Eq. (1), $\mathbf{x}_{j}=\left\{\mathbf{x}_{1, j}, \mathbf{x}_{2, j}, \mathbf{x}_{3, j}, \ldots, \mathbf{x}_{i, j}, \ldots, \mathbf{x}_{n, j}\right\}$ in each column, then get a new multidimensional time series.

\section{Appendix 2.}

According to the $\mathbf{x}_{j}$, then calculate the mean value in each row. Then, there is a new column:

$$
\mathbf{x}_{i}=\left\{\mathbf{x}_{1}, \mathbf{x}_{2}, \mathbf{x}_{3}, \ldots, \mathbf{x}_{i}, \ldots, \mathbf{x}_{n}\right\}, \quad \mathbf{x}_{i}=\operatorname{mean}\left(\mathbf{x}_{i, j}\right), j=1,2,3, \ldots
$$

\section{Appendix 3.}

According to Eq. (2), $\rho=\left[\rho_{(i, j)}\right], i, j=1,2, \ldots, n$, and $\left.\rho_{(i, j}\right)=x_{i}-x_{j}$,

$$
\|\rho\|_{2}=\sqrt{\lambda_{\max }\left(\rho^{*} \rho\right)}
$$

where $\rho^{*}$ is the conjugate transponce of $\rho$.

\section{Appendix 4 .}

To calculate the distance $(D)$ between two status matrices (initial status and considered status), the method used in this paper is

$$
D=\sqrt[4]{\left(\sum_{j=1} \rho_{(i, j) \text { initial }}-\sum_{j=1} \rho_{(i, j) \text { considered }}\right)^{2}\left(\sum_{i=1} \rho_{(i, j) \text { initial }}-\sum_{j=1} \rho_{(i, j) \text { considered }}\right)^{2}} .
$$

1. P. V. Marcal and I. P. King, "Elastic-plastic analysis of two-dimensional stress systems by the finite element method," Int. J. Mech. Sci., 9, No. 3, 143-155 (1967).

2. A. Borges and M. Peleg, "Determination of the apparent fractal dimension of the force-displacement curves of brittle snacks by four different algorithms," J. Texture Stud., 27, No. 3, 243-255 (1996).

3. J. Lemaitre, "How to use damage mechanics," Nucl. Eng. Des., 80, No. 2, 233-245 (1984).

4. J. Lemaitre, A Course on Damage Mechanics, Springer-Verlag, Berlin-Heidelberg (1996). 
5. J. L. Chaboche, "Continuum damage mechanics: Part II - Damage growth, crack initiation, and crack growth," J. Appl. Mech., 55, No. 1, 65-72 (1988).

6. G. Z. Voyiadjis, Handbook of Damage Mechanics, Springer, New York (2015).

7. T. H. G. Megson, Structural and Stress Analysis, Butterworth-Heinemann (2005).

8. R. E. Melchers and A. T. Beck, Structural Reliability Analysis and Prediction, John Wiley \& Sons (2017).

9. E. DiPasquale, J. W. Ju, A. Askar, and A. S. Çakmak, "Relation between global damage indices and local stiffness degradation," J. Struct. Eng., 116, No. 5, 14401456 (1990).

10. Y. Sawamoto, H. Tsubota, Y. Kasai, et al., "Analytical studies on local damage to reinforced concrete structures under impact loading by discrete element method," Nucl. Eng. Des., 179, No. 2, 157-177 (1998).

11. M. Jirásek and S. Marfia, "Non-local damage model based on displacement averaging," Int. J. Numer. Meth. Eng., 63, No. 1, 77-102 (2005).

12. E. Bannai and T. Ito, Algebraic Combinatorics I: Association Schemes, Benjamin/ Cummings, Menlo Park, CA (1984).

13. P. Flajolet and R. Sedgewick, Analytic Combinatorics, Cambridge University Press (2009).

14. P. van Overschee and B. L. de Moor, Subspace Identification for Linear Systems: Theory-Implementation-Applications, Springer Science \& Business Media (2012).

15. D. F. Andrews, "Plots of high-dimensional data," Biometrics, 28, No. 1, 125-136 (1972).

16. D. C. Lay, Linear Algebra and Its Applications (2010). 